\title{
Editorial
}

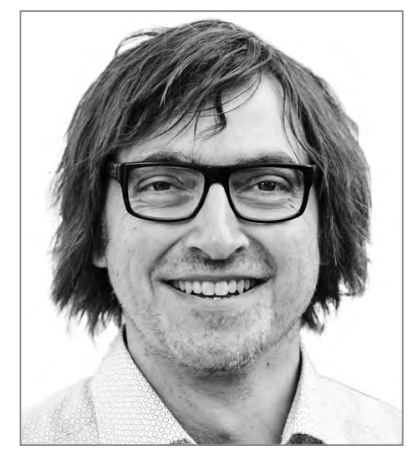

MARCEL WEIL

Institut für Technikfolgenabschätzung und Systemanalyse (ITAS), Karlsruhe (marcel.weil@kit.edu)
This is an article distributed under the terms of the Creative Commons Attribution License CCBY 4.0 (https:// creativecommons.org/licenses/by/4.0/) http://dx.doi.org/10.14512/tatup.27.3.3 m Herbst 2019 wird in Berlin das FUTURIUM eröffnet - ein Ort, an dem sich Menschen kreativ und auf sehr unterschiedliche Weise mit wichtigen Herausforderungen der Gegenwart und nahen Zukunft auseinandersetzen können. Hier werden Chancen und Risiken für die Gesellschaft sowie Möglichkeiten der Zukunftsgestaltung aufgezeigt - etwa in den Bereichen Ernährung, Gesundheit, Energie, Wohnen und Städte.

Zweifellos stellt die Erderwärmung, bzw. der Klimawandel eines der größten Risiken für die Zukunft der Menschheit dar. Vor kurzem hat der Weltklimarat IPCC in seinen Sonderbericht die notwendige Begrenzung der Erderwärmung auf $1,5^{\circ} \mathrm{C}$ (bis maximal $2^{\circ} \mathrm{C}$ ) nochmals unterstrichen. Bis $1,5^{\circ} \mathrm{C}$ werden die vielfältigen Folgen der Klimaerwärmung, wie z. B. die Zunahme von Extremwetterereignissen oder der Verlust von Biodiversität, als eher beherrschbar, oberhalb der Marke als weniger beherrschbar angesehen. Der Bericht zeigt aber auch, dass die 1,5-Grad-Marke bereits 2030 erreicht werden könnte, wenn nicht zeitnah massive Anstrengungen unternommen werden, dies zu verhindern. Unterblieben diese, so betonte jüngst UN-Generalsekretär António Guterres, dann ist die Menschheit existenziell bedroht.

Solche und ähnliche Apelle sind alles anderes als neu, sie gehen jedoch meist nach kurzen Nachrichten-Blitzlichtern im medialen Informationsrauschen unter. Tatsächlich haben wir in den vergangenen Jahren weltweit - trotz des scheinbar wachsenden Umweltbewusstseins - anstatt weniger immer mehr klimaschädliche Gase in die Atmosphäre des Planeten emittiert.

Es zeichnet sich deutlich ab, dass zur Erreichung der vereinbarten Klimaschutzziele von Paris (COP21) der alleinige Einsatz neuer und (wirklich) nachhaltiger Technologien nicht ausreichend ist. Vielmehr braucht es tiefgreifende Anstrengungen in allen Bereichen, die von Bevölkerung, Industrie und Staat mitgetragen werden müssen.

Einrichtungen wie das FUTURIUM können helfen, dass wichtige Themen wie der Klimawandel dauerhaft präsent sind und verhandelt werden.

Mögliche Fragen in diesem Rahmen lauten: Wer ist bereit, das hohe Risiko zu tragen, durch unser Verhalten das Klima soweit zu verändern, dass bereits in wenigen Jahren mit deutlichen und unumkehrbaren Folgen zu rechnen ist? Wer ist bereit, wichtige Veränderungen mit zu tragen, ja, aktiv zu unterstützen, auch wenn damit sogar Verzicht verbunden ist?

Dies ist ein wichtiger und notwendiger Aushandlungsprozess in der Gesellschaft. Hoffen wir, dass dieser nun an einer breiteren „Front“ und kontinuierlich geführt wird. Wir wünschen dem FUTURIUM hierfür und auch sonst alles Gute - und dem Gedanken, für das es steht.

\section{Marcel Weil}

